\title{
THE VIRTUES OF HESITATION: OPTIMAL TIMING IN A NON-STATIONARY WORLD ONLINE APPENDIX
}

\author{
URMEE KHAN AND MAXWELL B. STINCHCOMBE
}

We present versions of the first and second order condition (FOC and SOC) calculations as well as more complete arguments for the main results of 'The Virtues of Hesitation'.

\section{Optimal Timing for Cutting Off Search}

Model: at a flow cost of $c \geq 0$, one can keep searching for a source of higher profits. If found, expected net flow profits of $\bar{\pi}$ result. If one abandons the search, the decision is, by assumption, irreversible, and the known alternative yields expected net flow profits of $\underline{\pi}, \bar{\pi}>\underline{\pi}>0$. The infinitesimal analysis in "The Virtues of Hesitation" can be replaced by explicitly writing out the expected payoffs as integrals involving $t_{1}$, the time to cut off search, then setting the derivative to 0 and solving.

Let $C_{r}(w)=\int_{0}^{w} c e^{-r x} d x$ so that $\left[\frac{1}{r} \bar{\pi} e^{-r w}-C_{r}(w)\right]$ is the discounted net benefit if $W=w<t_{1}$ and $\left(1-F\left(t_{1}\right)\right)\left[\frac{1}{r} \underline{\pi} e^{-r t_{1}}-C_{r}\left(t_{1}\right)\right]$ is the expected discounted net benefit if

$W=w>t_{1}$. We will write out the first order conditions for the problem $\max _{t_{1} \in[0, \infty]} \psi\left(t_{1}\right)$ where

$$
\psi\left(t_{1}\right):=\int_{0}^{t_{1}}\left[\frac{1}{r} \bar{\pi} e^{-r w}-C_{r}(w)\right] f(w) d w+\left(1-F\left(t_{1}\right)\right)\left[\frac{1}{r} \underline{\pi} e^{-r t_{1}}-C_{r}\left(t_{1}\right)\right] .
$$

The first term in (1) is the integral over the event that $W<t_{1}$ of the discounted net benefit, and the second term covers the event $W>t_{1}$. Thus,

$$
\begin{aligned}
& \psi^{\prime}\left(t_{1}\right)=\left[\frac{1}{r} \bar{\pi} e^{-r t_{1}}-C_{r}\left(t_{1}\right)\right] f\left(t_{1}\right)- \\
& {\left[\frac{1}{r} \underline{\pi} e^{-r t_{1}}-C_{r}\left(t_{1}\right)\right] f\left(t_{1}\right)+\left(1-F\left(t_{1}\right)\right)\left[-\underline{\pi} e^{-r t_{1}}-C_{r}^{\prime}\left(t_{1}\right)\right] . }
\end{aligned}
$$

Now, $C_{r}^{\prime}\left(t_{1}\right)=c e^{-r t_{1}}$ and the $C_{r}\left(t_{1}\right) f\left(t_{1}\right)$ terms in (2) cancel, yielding

$$
\begin{aligned}
\psi^{\prime}\left(t_{1}\right) & =e^{-r t_{1}} f\left(t_{1}\right)\left[\frac{1}{r}(\bar{\pi}-\underline{\pi})\right]-\left(1-F\left(t_{1}\right)\right)[c+\underline{\pi}] \\
& =e^{-r t_{1}}\left(1-F\left(t_{1}\right)\right)\left[h\left(t_{1}\right) \frac{1}{r}(\bar{\pi}-\underline{\pi})-(c+\underline{\pi})\right] .
\end{aligned}
$$

Setting $\psi^{\prime}\left(t_{1}\right)=0$ yields the FOCs,

$$
h\left(t_{1}^{*}\right)=r(c+\underline{\pi}) /(\bar{\pi}-\underline{\pi}),
$$


Setting $m\left(t_{1}\right)=e^{-r t_{1}}\left(1-F\left(t_{1}\right)\right)$, the SOCs are

$$
\psi^{\prime \prime}\left(t_{1}^{*}\right)=m^{\prime}\left(t_{1}^{*}\right) \cdot 0+m\left(t_{1}^{*}\right) h^{\prime}\left(t_{1}^{*}\right)[\bar{\pi}-\underline{\pi}]<0,
$$

which can only be satisfied if $h^{\prime}\left(t_{1}^{*}\right)<0$ because $m\left(t_{1}\right)>0$ when $h\left(t_{1}\right)>0$.

\section{Optimal Timing of InNoculation}

Model: present utility flows are $\bar{u}>0$. At issue is the optimal timing of a costly innoculation or precautionary measure. If the measure is not in place before $W$, then $\bar{u}$ will be reduced to a utility flow normalized to 0 . If the measure is in place, $W$ will reduce utility flows to $\underline{u}$, with $\bar{u}>\underline{u}>0$. As well as reducing the damages in case of crisis, innoculation at time $t_{1}$ reduces the probability of a crisis by changing the arrival density from $f(w)$ to

$$
f_{\theta}\left(w ; t_{1}\right)= \begin{cases}f(w) & \text { if } w<t_{1} \\ (1-\theta) f(w) & \text { if } w \geq t_{1} .\end{cases}
$$

After the innoculation, $\theta \int_{t_{1}}^{\infty} f(w) d w$ of the probability shifts to the mass point at infinity, that is, to the disaster not arriving.

We first give a detailed account of the roundabout method of arriving at the first order conditions, then turn to a brief analysis of the post-innoculation hazard rate.

2.1. Roundabout Calculations. Again, we will write out $\psi\left(t_{1}\right)$, the expected net benefits of waiting until $t_{1}$, in this case. The expected cost term is similar to the term in the analysis of the optimal delay to cutting off search, but the expected benefits terms must cover three events, $W<t_{1}, t_{1}<W<\infty$, and $W=\infty$.

2.1.1. Expected Costs. If $t_{1}$ is the decision time for innoculation, the contribution of expected discounted costs to utility are $-\left(1-F\left(t_{1}\right)\right) C e^{-r t_{1}}$. The derivative with respect to $t_{1}$ is therefore

$$
\frac{d(\text { Costs })}{d t_{1}}=C e^{-r t_{1}} f\left(t_{1}\right)+r C e^{-r t_{1}}\left(1-F\left(t_{1}\right)\right)
$$

2.1.2. Expected Flow Benefits. For the effect of the choice of $t_{1}$ on the expected utility flows, we first consider three types of events, then give the expected flow payoffs associated with each event, then give their expected values and their derivatives with respect to $t_{1}$.

Three types of events

There are three types of events with probability affected by the choice of $t_{1}$,

(A) $W=w$ and $0 \leq w<t_{1}$,

(B) $W=w$ and $t_{1} \leq w<\infty$,

(C) $W=w$ and $w=\infty$. 
If (A) happens, payoffs are $\int_{0}^{w} \bar{u} e^{-r x} d x+\int_{w}^{\infty} 0 e^{-r x} d x=\int_{0}^{w} \bar{u} e^{-r x} d x$.

If (B) happens, payoffs are $\int_{0}^{w} \bar{u} e^{-r x} d x+\int_{w}^{\infty} \underline{u} e^{-r x} d x$.

If (C) happens, payoffs are $\int_{0}^{\infty} \bar{u} e^{-r x} d x$.

Expected flow payoffs and their derivatives

Integrating over case (A), the expected flow payoffs are

$$
\int_{0}^{t_{1}}\left[\int_{0}^{w} \bar{u} e^{-r x} d x\right] f(w) d w=\int_{0}^{t_{1}} \bar{u} \frac{1}{r}\left(1-e^{-r w}\right) f(w) d w
$$

so that $a 1:=d(\mathrm{~A}) / d t_{1}$ satisfies

$$
a 1=\bar{u} \frac{1}{r}\left(1-e^{-r t_{1}}\right) f\left(t_{1}\right) .
$$

Integrating over case (B), the expected flow payoffs are

$$
\begin{gathered}
\int_{t_{1}}^{\infty}\left[\int_{0}^{w} \bar{u} e^{-r x} d x f(w) d w+\int_{w}^{\infty} \underline{u} e^{-r x} d x\right](1-\theta) f(w) d w= \\
\int_{t_{1}}^{\infty}\left[\bar{u} \frac{1}{r}\left(1-e^{-r w}\right)+\underline{u} \frac{1}{r} e^{-r w}\right](1-\theta) f(w) d w
\end{gathered}
$$

so that $b 1:=d(\mathrm{~B}) / d t_{1}$ satisfies

$$
b 1=-\left[\bar{u} \frac{1}{r}\left(1-e^{-r t_{1}}\right)+\underline{u} \frac{1}{r} e^{-r t_{1}}\right](1-\theta) f\left(t_{1}\right) .
$$

Integrating over case $(\mathrm{C})$, the expected flow payoffs are

$$
\left[q+\theta \int_{t_{1}}^{\infty} f(w) d w\right] \int_{0}^{\infty} \bar{u} e^{-r x} d x=\left[q+\theta \int_{t_{1}}^{\infty} f(w) d w\right] \bar{u} \frac{1}{r}
$$

where $q=P(W=\infty)$. With $c 1:=d(\mathrm{C}) / d t_{1}$, this yields

$$
c 1=-\bar{u} \frac{1}{r} \theta f\left(t_{1}\right) \text {. }
$$

2.1.3. First order conditions. The FOCs are $\psi^{\prime}\left(t_{1}\right)=a 1+b 1+c 1+d($ Costs $) / d t_{1}=0$ where

$$
\begin{aligned}
a 1 & =\bar{u} \frac{1}{r}\left(1-e^{-r t_{1}}\right) f\left(t_{1}\right) \\
b 1 & =-\left[\bar{u} \frac{1}{r}\left(1-e^{-r t_{1}}\right)+\underline{u} \frac{1}{r} e^{-r t_{1}}\right](1-\theta) f\left(t_{1}\right) \\
c 1 & =-\bar{u} \frac{1}{r} \theta f\left(t_{1}\right) \\
\frac{d(\text { Costs })}{d t_{1}} & =C e^{-r t_{1}} f\left(t_{1}\right)+r C e^{-r t_{1}}\left(1-F\left(t_{1}\right)\right) .
\end{aligned}
$$

Gathering the terms with the common factor $f\left(t_{1}\right)$ yields

$$
f\left(t_{1}\right)\left[\bar{u} \frac{1}{r}\left(1-e^{-r t_{1}}\right)-\bar{u} \frac{1}{r}\left(1-e^{-r t_{1}}\right)(1-\theta)+\underline{u} \frac{1}{r} e^{-r t_{1}}(1-\theta)-\bar{u} \frac{1}{r} \theta+C e^{-r t_{1}}\right],
$$

which simplifies to

$$
f\left(t_{1}\right)\left[\bar{u} \frac{1}{r}\left\{\left(1-e^{-r t_{1}}\right) \theta-\theta\right\}+\underline{u} \frac{1}{r} e^{-r t_{1}}(1-\theta)+C e^{-r t_{1}}\right] .
$$


Simplifying $\left\{\left(1-e^{-r t_{1}}\right) \theta-\theta\right\}$ to $-e^{-r t_{1}} \theta$ and taking out the common factor of $e^{-r t_{1}}$ yields

$$
f\left(t_{1}\right) e^{-r t_{1}}\left[-\bar{u} \frac{1}{r} \theta+\underline{u} \frac{1}{r}(1-\theta)+C\right] .
$$

Adding the second part of the cost term, $r C e^{-r t_{1}}\left(1-F\left(t_{1}\right)\right)$, setting the resultant sum equal to 0 , and removing the factor of $e^{-r t_{1}}\left(1-F\left(t_{1}\right)\right)$ yields

$$
\psi^{\prime}\left(t_{1}\right)=e^{-r t_{1}}\left(1-F\left(t_{1}\right)\right)\left[h\left(t_{1}\right)\left(-\bar{u} \frac{1}{r} \theta+\underline{u} \frac{1}{r}(1-\theta)+C\right)+r C\right] .
$$

Setting $\psi^{\prime}\left(t_{1}^{*}\right)=0$, the FOCs are

$$
h\left(t_{1}^{*}\right)=\frac{r C}{\frac{1}{r}(\theta \bar{u}+(1-\theta) \underline{u})-C},
$$

and the SOCs are

$$
\psi^{\prime \prime}\left(t_{1}^{*}\right)=m^{\prime}\left(t_{1}^{*}\right) \cdot 0+m\left(t_{1}^{*}\right)\left[h^{\prime}\left(t_{1}\right)\left(-\bar{u} \frac{1}{r} \theta+\underline{u} \frac{1}{r}(1-\theta)+C\right)+r C\right]<0,
$$

and these can only be satisfied if $h^{\prime}\left(t_{1}^{*}\right)>0$ because $m\left(t_{1}\right)>0$ when $h\left(t_{1}\right)>0$.

2.2. Some Details About the Post-Innoculation Hazard Rate. The hazard rate at $t^{\prime}>t_{1}$ is

$$
h_{c}\left(t^{\prime}\right)=\frac{(1-\theta) f\left(t^{\prime}\right)}{\left(1-\left[F\left(t_{1}\right)+(1-\theta) \int_{t_{1}}^{t^{\prime}} f(x) d x\right]\right)} .
$$

Provided the density stays positive, $h_{c}\left(t^{\prime}\right)<(1-\theta) h\left(t^{\prime}\right)$ for all $t^{\prime}>t_{1}$ because the numerator goes down by a larger factor than the denominator. In particular, for a second change of action at some time $t_{2}>t_{1}$, one must be using $h_{c}\left(t_{2}\right)$, and this is not $(1-\theta) h\left(t_{2}\right)$. However, close to $t_{1}$, this is a good approximation because $\lim _{t^{\prime} \downarrow t_{1}} h_{c}\left(t^{\prime}\right)=(1-\theta) h\left(t_{1}\right)$.

\section{Details of the Bellman Equation Proof}

We here give the details of the proof of Theorem 1, the Bellman equation result. For ease of reference, the result is re-stated below after a quick review.

1. Change costs: changing action from $a$ to $b$ in state $i$ costs, $c(a, b ; i)$ where $c:(A \times$ A) $\times S \rightarrow \mathbb{R}_{++}$is continuous, has a strictly positive minimum value, and has the property that direct changes are always cheaper than multi-step changes of action, $c\left(a, a^{\prime} ; i\right)+c\left(a^{\prime}, a^{\prime \prime} ; i\right)>c\left(a, a^{\prime \prime} ; i\right)$.

2. Flow utilities are given by a continuous $u: S \times A \rightarrow \mathbb{R}$ and are continuously discounted at rate $r$. 
3. The within interval expected reward to using $\mathfrak{p}$ from $(i, a)$ is denoted $\mathcal{R}(\mathfrak{p} ;(i, a))$ and combines flow payoffs with change costs as follows,

$$
\begin{aligned}
\mathcal{R}(\mathfrak{p} ;(i, a))= & \int_{0}^{\infty}\left[\int_{0}^{s} u(i, \mathfrak{p}(t)) e^{-r t} d t\right] f(s ; \mathfrak{p}, i) d s \\
& +\int_{0}^{\infty}\left[\sum_{t \in T_{\mathfrak{p}}, t<s} c(\mathfrak{p}(t), \mathfrak{p}(t+) ; i) e^{-r t}\right] f(s ; \mathfrak{p}, i) d s .
\end{aligned}
$$

4. $\nu_{\mathfrak{p}}$ denotes the distribution, possibly incomplete, random waiting-time and state pair associated with $\mathfrak{p}$.

5. $V^{*}$ denotes the value function for the sMdp.

Theorem 1. For an $s M d p$, the mapping $T: C(S \times A) \rightarrow C(S \times A)$ defined by

$$
T(V)(i, a)=\max _{\mathfrak{p} \in \mathbb{P}(i, a)}\left[\mathcal{R}(\mathfrak{p} ;(i, a))+\int_{(0, \infty) \times S} V(i, \mathfrak{p}(s)) e^{-r s} d \nu_{\mathfrak{p}}(s, i)\right]
$$

has contraction factor at most $\beta:=\sup _{(i, a), \mathfrak{p} \in \mathbb{P}(i, a)} \int_{0}^{\infty} e^{-r s} f(s ; \mathfrak{p}, i) d s<1$, its unique fixed point is the value function, $V^{*}$, and following the complete semi-stationary plan given by the argmax policy for (23) in every stochastic interval delivers expected payoffs $V^{*}(i, a)$ for every $(i, a) \in S \times A$.

3.1. Preliminaries to the Proof. In the proof, we make appeal to the Theorem of the Maximum to study the problem

$$
\max _{\mathfrak{p} \in \mathbb{P}(i, a)}\left[\mathcal{R}(\mathfrak{p} ;(i, a))+\int_{(0, \infty) \times S} V(i, \mathfrak{p}(s)) e^{-r s} d \nu_{\mathfrak{p}}(s, i)\right] .
$$

Given the assumption that $(i, a) \mapsto \mathbb{P}(i, a)$ is continuous and compact valued, we need only prove the joint continuity of the mapping

$$
(\mathfrak{p},(i, a)) \mapsto\left[\mathcal{R}(\mathfrak{p} ;(i, a))+\int_{(0, \infty) \times S} V(i, \mathfrak{p}(s)) e^{-r s} d \nu_{\mathfrak{p}}(s, i)\right] .
$$

3.1.1. Metrics. In these results, crucial roles are played by the metric, $d_{H}$, on plans and a tractable class of $d_{H}$-compact sets of plans as subsets of $[0, \infty) \times A$.

The metric $D(\cdot, \cdot)$ on $[0, \infty) \times A$

The set $A$ is, by assumption, compact. If it is a compact subset of a vector space with metric $r$, e.g. $\mathbb{R}^{\ell}$ with $r(x, y)=\|x-y\|$, then the metric is $[0, \infty) \times A$ is defined by

$$
D\left((t, a),\left(t^{\prime}, a^{\prime}\right)\right)=\left|e^{-\rho t}-e^{-\rho t^{\prime}}\right|+r\left(e^{-\rho t} a, e^{-\rho t^{\prime}} a^{\prime}\right)
$$

for some $\rho>0$. To make this work in full generality, we homeomorphically imbed $A$ as a subset of a metric vector space. 
Let $\mathbb{M}$ be the subset of $\mathbb{R}^{\mathbb{N}}$ for which $\sum_{n}\left|x_{n}\right| / 2^{n}$ is finite and give $\mathbb{M}$ the $\ell_{1}$-metric $r(x, y)=\sum_{n}\left|x_{n}-y_{n}\right| / 2^{n}$. Let $\left\{e_{n}: n \in \mathbb{N}\right\}$ be a dense subset of the compact metric space $A$ and define the bijection between $A$ by $\varphi(a)=\left\{D_{A}\left(a, e_{n}\right): n \in \mathbb{N}\right\}$. Since $\lim _{\alpha \rightarrow \infty} D_{A}\left(a_{\alpha}, a\right)=0$ iff $\lim _{\alpha \rightarrow \infty} r\left(\varphi\left(a_{\alpha}\right), \varphi(a)\right) \rightarrow 0, \varphi(\cdot)$ is a homeomorphism so that $\varphi(A)$ is $r$-compact.

We define the metric $D$ on $[0, \infty) \times A$ by

$$
D\left((t, a),\left(t^{\prime}, a^{\prime}\right)\right)=\left|e^{-\rho t}-e^{-\rho t^{\prime}}\right|+r\left(e^{-\rho t} \varphi(a), e^{-\rho t^{\prime}} \varphi\left(a^{\prime}\right)\right)
$$

for some $\rho>0$. The following Lemma tells us that differences in the actions at later times make a vanishingly small difference if they happen at later points in time. This property will play a crucial role in showing that there is no loss in restricting attention to a compact set of plans.

Lemma 1. $\max _{a, a^{\prime} \in A, t, t^{\prime} \geq T} D\left((t, a),\left(t^{\prime}, a^{\prime}\right)\right) \rightarrow 0$ as $T \uparrow \infty$.

Proof. $r\left(e^{-\rho t} \varphi(a), e^{-\rho t^{\prime}} \varphi\left(a^{\prime}\right)\right)$ is less than or equal to $r\left(e^{-\rho t} \varphi(a), 0\right)+r\left(0, e^{-\rho t^{\prime}} \varphi\left(a^{\prime}\right)\right)$ and $\max _{a \in A} r\left(e^{-\rho t} \varphi(a), 0\right)$ decreases to 0 as $t \uparrow \infty$.

The metric $d_{H}(\cdot, \cdot)$ on $\mathbb{P}$

Define the distance between $\mathfrak{p} \in \mathbb{P}(i, a)$ and $\mathfrak{p}^{\prime} \in \mathbb{P}\left(i^{\prime}, a^{\prime}\right)$ by

$$
d_{H}\left(\mathfrak{p}, \mathfrak{p}^{\prime}\right)=\inf \left\{\epsilon>0: \operatorname{gr}(\mathfrak{p}) \subset g r\left(\mathfrak{p}^{\prime}\right)^{\epsilon} \text { and } \operatorname{gr}\left(\mathfrak{p}^{\prime}\right) \subset g r(\mathfrak{p})^{\epsilon}\right\}
$$

where $\operatorname{gr}(\mathfrak{p})$ and $\operatorname{gr}\left(\mathfrak{p}^{\prime}\right)$ are the graphs of the plans while $\operatorname{gr}(\mathfrak{p})^{\epsilon}$ and $g r\left(\mathfrak{p}^{\prime}\right)^{\epsilon}$ are the sets of points at $D$-distance less than $\epsilon$ from the graphs.

Lemma 1 implies that for any $\epsilon>0$, there exists a $T<\infty$ such that any difference between plans on $[T, \infty)$ makes a $d_{H}$-distance difference of less than $\epsilon$.

3.1.2. Compact Sets of Plans. We have assumed that the correspondence $(i, a) \mapsto \mathbb{P}(i, a)$ is continuous and compact-valued. To simplify matters, we have also assumed that the number of planned changed times in all of the sets $\mathbb{P}(i, a)$ is uniformly bounded. This has the property that the discounted costs of action changes, $\sum_{t \in T_{\mathfrak{p}}} e^{-r t}$, is bounded, a crucial step in the proof of the joint continuity result, Lemma 3. There are a number of alternative ways to achieve this.

- The Lemma below shows that assuming the existence of a uniform bound, $\tau>0$, to the time between successive planned action change times delivers a compact set of plans. As this implies that there are at most $1+T / \tau$ actions changes by any time $T$, we again have the crucial property that $\sum_{t \in T_{\mathfrak{p}}} e^{-r t}$, is bounded.

- To avoid a lower bound between time changes, we could have complicated the space of plans to allow successive action change times to happen at 0 formal delays but to still record the order of the changes (as in Simon and Stinchcombe (1989) or Stinchcombe (1992)). 
With this in place, we could have restricted attention to the compact sets plans defined by the property that $\sum_{t \in T_{\mathfrak{p}}} e^{-r t}$ is uniformly bounded.

For $\tau>0$, let $\mathbb{K}_{\tau}(i, a)$ denote the set of all plans starting at $(i, a)$ such with action change times at least $\tau$ apart, that is, if $0 \leq t_{k}<t_{k+1}$ are action change times, then $t_{k+1}-t_{k} \geq \tau$. Since the first change of action can happen at $t=0$, for any time $t \geq 0$, the number of action change times for any plan in $\mathbb{K}_{\tau}(i, a)$ is bounded above by $1+t / \tau$.

Lemma 2. For $(i, a) \in S \times A$ and $\tau>0, \mathbb{K}_{\tau}(i, a)$ is $d_{H}$-compact and the correspondence $(i, a) \mapsto \mathbb{K}_{\tau}(i, a)$ is continuous.

Proof. Given compactness, the continuity of the correspondence is immediate because $a$ determines the starting action of the set of plans.

Let $\mathfrak{p}_{\alpha}, \alpha \in \mathbb{N}$, be a sequence of plans in $\mathbb{K}_{\tau}(i, a)$ having change times and actions $\left(t_{1, \alpha}, a_{1, \alpha}\right), \ldots,\left(t_{K_{\alpha}, \alpha}, a_{K_{\alpha}, \alpha}\right), K_{\alpha} \in \mathbb{N} \cup\{\infty\}$. Using Cantor's diagonalization procedure, (Corbae, Stinchcombe and Zeman, 2009, p. 61) there exists a subsequence, still denoted $\mathfrak{p}_{\alpha}$, with $K_{\alpha}$ convergent to some point, $K$, in $\mathbb{N} \cup\{\infty\}$, each $t_{k, \alpha}$ convergent to some $t_{k} \in[0, \infty]$, $k \leq K$, and each $a_{k, \alpha}$ convergent to some $a_{k} \in A$. We must identify a limit plan $\mathfrak{p}_{\infty}$ and show that $d_{H}\left(\mathfrak{p}_{\alpha}, \mathfrak{p}_{\infty}\right) \rightarrow 0$.

If $\left\{k \leq K: t_{k}=\infty\right\}$ is empty, define $\mathfrak{p}_{\infty}$ as the one changing to $a_{k}$ at $t_{k}, k=1, \ldots, K$, if $\left\{k \leq K: t_{k}=\infty\right\}$ is not empty, let $K^{\circ}$ denote its minimal element and define $\mathfrak{p}_{\infty}$ as changing to $a_{k}$ at $t_{k}$ for $k=1, \ldots, K^{\circ}-1$. The change times in $\mathfrak{p}_{\infty}$ satisfy $t_{k}+\tau \leq t_{k+1}$ because $t_{k, \alpha}+\tau \leq t_{k+1, \alpha}$ for all $\alpha, t_{k, \alpha} \rightarrow t_{k}$, and $t_{k+1, \alpha} \rightarrow t_{k+1}$. Thus, $\mathfrak{p}_{\infty} \in \mathbb{K}_{\tau}(i, a)$. Finally, $d_{H}\left(\mathfrak{p}_{\alpha}, \mathfrak{p}_{\infty}\right) \rightarrow 0$ because the convergence of change times and actions is uniform over any time interval $[0, T]$ (because there are at most $1+T / \tau$ change times), and, by Lemma 1 , differences on $[T, \infty)$ matter less than any $\epsilon>0$ for large enough $T$.

3.1.3. Joint Continuity. The joint continuity of the maximand in the following allows us to apply the Theorem of the Maximum to the problem in Theorem 1. The proof involves, essentially, repeated use of a basic $L^{1}-L^{\infty}$ duality result, re-proved here for completeness.

Lemma 3. For $\tau>0,(\mathfrak{p},(i, a)) \mapsto\left[\mathcal{R}(\mathfrak{p} ;(i, a))+\int_{(0, \infty) \times S} V(j, \mathfrak{p}(s)) e^{-r s} d \nu_{\mathfrak{p}}(s, j)\right]$ is jointly continuous on the graph of the correpondence $(i, a) \mapsto \mathbb{K}_{\tau}(i, a)$.

Proof. Let $\left(\mathfrak{p}_{\alpha},\left(i_{\alpha}, a_{\alpha}\right)\right), \alpha \in \mathbb{N}$, be a sequence with $\mathfrak{p}_{\alpha} \in \mathbb{K}_{\tau}\left(i_{\alpha}, a_{\alpha}\right)$ and suppose that $\left(\mathfrak{p}_{\alpha},\left(i_{\alpha}, a_{\alpha}\right)\right) \rightarrow(\mathfrak{p},(i, a))$. Let $f_{\alpha}$ denote the density $f\left(\cdot ; \mathfrak{p}_{\alpha}, i_{\alpha}\right)$ and $f$ the density $f(\cdot ; \mathfrak{p}, i)$. By $L_{1}$-continuity, $\int\left|f_{\alpha}(s)-f(s)\right| d s \rightarrow 0$. We will use the following claim.

A Duality Claim: If $h$ and $\left\{h_{\alpha}: \alpha \in \mathbb{N}\right\}$ are a uniformly bounded set of measurable functions with the property that for all $\epsilon>0$ and all $T>0$, there exists an $\alpha^{\prime}$ such that for all $\alpha>\alpha^{\prime}, \lambda\left(\left\{t \in[0, T]:\left|h_{\alpha}(t)-h(t)\right|>\epsilon\right\}\right)<\epsilon$, then

$$
\int_{0}^{\infty} h_{\alpha}(s) f_{\alpha}(s) d s \rightarrow \int_{0}^{\infty} h(s) f(s) d s .
$$


Note that $\left|\int_{0}^{\infty} h_{\alpha}(s) f_{\alpha}(s) d s-\int_{0}^{\infty} h(s) f(s) d s\right| \leq \int_{0}^{\infty}\left|h_{\alpha}(s) f_{\alpha}(s)-h(s) f(s)\right| d s$, and for each $s \in[0, \infty)$,

$$
\left|h_{\alpha}(s) f_{\alpha}(s)-h(s) f(s)\right| \leq\left|h_{\alpha}(s) f_{\alpha}(s)-h_{\alpha}(s) f(s)\right|+\left|h_{\alpha}(s) f(s)-h(s) f(s)\right| .
$$

For the claim, it is sufficient to show that $\int_{0}^{\infty}\left|h_{\alpha}(s) f_{\alpha}(s)-h_{\alpha}(s) f(s)\right| d s \rightarrow 0$ and $\int_{0}^{\infty} \mid h_{\alpha}(s) f(s)-$ $h(s) f(s) \mid d s \rightarrow 0$.

For the first, note that $\left|h_{\alpha}(s) f_{\alpha}(s)-h_{\alpha}(s) f(s)\right|=\left|h_{\alpha}(s)\right| \cdot\left|f_{\alpha}(s)-f(s)\right| \leq B \cdot\left|f_{\alpha}(s)-f(s)\right|$ where $B$ is a uniform bound for $\left\{h_{\alpha}: \alpha \in \mathbb{N}\right\}$. This yields

$$
\int_{0}^{\infty}\left|h_{\alpha}(s) f_{\alpha}(s)-h_{\alpha}(s) f(s)\right| d s \leq B \cdot \int_{0}^{\infty}\left|f_{\alpha}(s)-f(s)\right| d s \rightarrow 0 .
$$

For the second, note that $\left|h_{\alpha}(s) f(s)-h(s) f(s)\right|=\left|h_{\alpha}(s)-h(s)\right| \cdot f(s)$. Now, pick $T$ such that $\int_{T}^{\infty} f(s) d s<\epsilon / 2 B$ where $B$ is a uniform bound for $h$ and $\left\{h_{\alpha}: \alpha \in \mathbb{N}\right\}$. This yields

$$
\begin{aligned}
\int_{0}^{\infty} \mid h_{\alpha}(s)- & h(s) \mid f(s) d s \\
= & \int_{0}^{T}\left|h_{\alpha}(s)-h(s)\right| f(s) d s+\int_{T}^{\infty}\left|h_{\alpha}(s)-h(s)\right| f(s) d s \\
& \leq \int_{0}^{T}\left|h_{\alpha}(s)-h(s)\right| f(s) d s+\epsilon .
\end{aligned}
$$

Since $\epsilon$ was arbitrary, to establish the claim, it is sufficient to show that the first term in (34) converges to 0 for any $T$. For any subsequence of the $h_{\alpha}$, there is a further subsequence such that $\left|h_{\alpha}(s)-h(s)\right| f(s) \rightarrow 0$ for $\lambda$-almost all $s \in[0, T]$. By dominated convergence, this implies that $\int_{0}^{T}\left|h_{\alpha}(s)-h(s)\right| f(s) d s \rightarrow 0$, establishing the claim.

Returning to the proof of the joint continuity, there are three parts to the function $(\mathfrak{p} ;(i, a)) \mapsto\left[\mathcal{R}(\mathfrak{p} ;(i, a))+\int_{(0, \infty) \times S} V(i, \mathfrak{p}(s)) e^{-r s} d \nu_{\mathfrak{p}}(s, i)\right]$, the flow payoff part of and the costs part of $\mathcal{R}(\cdot)$, and the integrated expected value of $V(\cdot, \cdot)$.

For the flow payoffs, define the uniformly bounded sequence of (Lipschitz) functions $h_{\alpha}$ by $h_{\alpha}(s)=\int_{0}^{s} u\left(i, \mathfrak{p}_{\alpha}(t)\right) e^{-r t} d t$ and define $h(s)=\int_{0}^{s} u(i, \mathfrak{p}(t)) e^{-r t} d t$. The $h_{\alpha}$ converge uniformly to $h$, hence $\int h_{\alpha}(s) f_{\alpha}(s) d s \rightarrow \int h(s) f(s) d s$ by the Claim.

For the costs part, define $g_{\alpha}(s)=\sum_{t \in T_{\mathfrak{p}_{\alpha}}, t<s} c\left(\mathfrak{p}_{\alpha}(t), \mathfrak{p}_{\alpha}(t+) ; i_{\alpha}\right) e^{-r t}$ and $g(s)$ the corresponding function for $(\mathfrak{p},(i, a))$. The sequence of functions $g_{\alpha}$ is uniformly bounded because, by any time $t$ in $\mathbb{K}_{\tau}(i, a)$, there are at most $1+t / \tau$ action changes and costs incurred at $t$ are discounted by $e^{-r t}$. By the joint continuity of $c(\cdot, \cdot ; \cdot)$ and $f(\cdot ; \cdot, \cdot)$, the functions $g$ and $\left\{g_{\alpha}: \alpha \in \mathbb{N}\right\}$ satisfy the conditions of the Claim, hence $\int g_{\alpha}(s) f_{\alpha}(s) d s \rightarrow \int g(s) f(s) d s$.

Finally, we must show that $\int_{(0, \infty) \times S} V\left(j, \mathfrak{p}_{\alpha}(s)\right) e^{-r s} d \nu_{\mathfrak{p}_{\alpha}}(s, j)$ converges. Recall that for $E \times F \subset(0, \infty) \times S$ and plan $\mathfrak{p}_{\alpha} \in \mathbb{P}\left(i_{\alpha}, a_{\alpha}\right), \nu_{\mathfrak{p}_{\alpha}}(E \times F)$ is defined as $\int_{E} f\left(s ; \mathfrak{p}_{\alpha}, i_{\alpha}\right)$. $\mu\left(s ; \mathfrak{p}_{\alpha}, i_{\alpha}\right)(F) d s$. Because $S$ and $A$ are compact and $V(\cdot, \cdot)$ is jointly continuous, as $a_{\alpha} \rightarrow$ $a$, the functions $i \mapsto V\left(i, a_{\alpha}\right)$ converge uniformly to the function $i \mapsto V(i, a)$. The joint 
continuity of $\mu(\cdot ; \cdot, \cdot)$ therefore implies that the continuous function

$$
s \mapsto m_{\alpha}(s):=\int_{S} V\left(j, a_{\alpha}\right) e^{-r s} \mu\left(s ; \mathfrak{p}_{\alpha}, i_{\alpha}\right)(d j)
$$

converges to the continuous function

$$
s \mapsto m(s):=\int_{S} V(j, a) e^{-r s} \mu(s ; \mathfrak{p}, i)(d j) .
$$

Further, joint continuity implies that convergence is uniform over any bounded set of $s$, and since the function is uniformly less than any $\epsilon>0$ for all $s \geq T$ for some $T<\infty$, the convergence is in fact uniform over $(0, \infty)$. By the Claim given above, $\int_{(0, \infty)} m_{\alpha}(s) f_{\alpha}(s) d s \rightarrow \int_{(0, \infty)} m(s) f(s) d s$.

We are now in a position to give the following.

Proof of Theorem 1: In outline, we show (1) the maping $T$ in the optimization problem (23) is well-defined, (2) that has the given contraction factor, (3) that the unique fixed point of $T$ is the true value function, $V^{*}$, and (4) that following the argmax policy in (23) delivers $V^{*}$.

(1) By Berge's theorem of the maximum, the assumed continuity of the correspondence and the joint continuity of Lemma 3 imply that the mapping $T$ in the optimization problem (23) is well-defined. ${ }^{1}$

(2) From Blackwell's Lemma for contraction mappings (e.g. (Corbae, Stinchcombe and Zeman, 2009, Lemma 6.2.33)), to show that $T$ is a contraction mapping with contraction factor $\beta$, it is sufficient to show that (a) $T$ is monotonic, and (b) that for any constant $\kappa \geq 0$ and $V \in C(S \times A), T(V+\kappa) \leq T(V)+\beta \kappa$. Monotonicity is immediate. For (b), define $\Psi(V, \mathfrak{p})=\left[\mathcal{R}(\mathfrak{p} ;(i, a))+\int_{(0, \infty) \times S} V(i, \mathfrak{p}(s)) e^{-r s} d \nu_{\mathfrak{p}}(s, i)\right]$, let $\mathfrak{p}^{*}$ and $\mathfrak{p}^{\kappa}$ solve

$$
\max _{\mathfrak{p} \in \mathbb{P}(i, a)} \Psi(V, \mathfrak{p}) \text { and } \max _{\mathfrak{p} \in \mathbb{P}(i, a)} \Psi(V+\kappa, \mathfrak{p})
$$

so that $\Psi\left(V, \mathfrak{p}^{*}\right)=T(V)$ and $\Psi\left(V+k, \mathfrak{p}^{\kappa}\right)=T(V+\kappa)$. By the optimality of $\mathfrak{p}^{\kappa}$, $\Psi\left(V+\kappa, \mathfrak{p}^{*}\right) \leq \Psi\left(V+\kappa, \mathfrak{p}^{\kappa}\right)$. Further $\Psi\left(V+\kappa, \mathfrak{p}^{*}\right)=\Psi(V)+\int_{(0, \infty) \times S} \kappa e^{-r s} d \nu_{\mathfrak{p}^{*}}(s, i)$ and $\int_{(0, \infty) \times S} \kappa e^{-r s} d \nu_{\mathfrak{p}^{*}}(s, i) \leq \kappa \sup _{(i, a), \mathfrak{p} \in \mathbb{P}(i, a)} e^{-r s} f(s ; \mathfrak{p}, i) d s=\kappa \cdot \beta$. By $L^{1}$-continuity and compactness, the supremum defining $\beta$ is achieved, and since waiting times are all strictly positive, $\beta<1$.

(3) and (4) The arguments parallel the standard discrete-time dynamic programming arguments: if $V_{\dagger}$ is the unique fixed point for $T$, then following the $\operatorname{argmax} \mathfrak{p}^{*}$ for the first stochastic interval and receiving $V_{\dagger}\left(I_{1}, a\right)$ when the interval ends must have value $V_{\dagger}$. Therefore following $\mathfrak{p}^{*}$ for the first two stochastic intervals has value $V_{\dagger}$. Since the strictly positive $W_{k}$ have expectation uniformly bounded away from 0 (by uniform smoothness),

\footnotetext{
${ }^{1}$ Berge's theorem of the maximum is usually stated with joint continuity, not joint continuity on the graph of the correspondence. However, the graph is a closed subset of a metric space and the Tietze-Urysohn extension theorem, e.g. Dudley (2002, Theorem 2.6.4), tells us that continuous $\mathbb{R}$-valued functions on closed subsets of metric spaces have continuous extensions.
} 
following $\mathfrak{p}^{*}$ in each interval yields $V_{\dagger}$. By definition, $V^{*} \geq V_{\dagger}$, and if $V^{*}\left(i_{0}, a_{0}\right)>V_{\dagger}\left(i_{0}, a_{0}\right)$ for some $\left(i_{0}, a_{0}\right)$, then $V_{\dagger}$ is not the fixed point of $T$.

\section{Alternative Derivation of Euler Equations}

We now present a rather roundabout argument for the Euler equation result, the FOCs in Theorem 2. These involve only the value function and aspects of stochastic structure local to $t_{1}^{*}$. Without the assumption that the stochastic structure is localized, there are two additional terms involving integrated discounted future partial derivatives, decidedly nonlocal quantities. To put it another way, under the simplifying assumptions of Theorem 2 , future considerations enter the local analysis of the optimality of a $t_{1}^{*}$ only through the value function and local properties of the stochastic structure, while, if we abandon these assumptions, the non-local properties of the stochastic structure as well as the value function must be considered.

Theorem 2. In an sMdp with a localized stochastic structure, if $\mathfrak{p}^{*}$ solves,

$$
\max _{\mathfrak{p} \in \mathbb{P}\left(i_{0}, a_{0}\right)}\left[\mathcal{R}\left(\mathfrak{p} ;\left(i_{0}, a_{0}\right)\right)+\int_{(0, \infty) \times S} V^{*}(i, \mathfrak{p}(s)) e^{-r s} d \nu_{\mathfrak{p}}(s, i)\right]
$$

hesitates until a time $t_{1}^{*} \in(0, \infty)$ for its single action change to $a_{1}^{*}$ at cost $c^{*}$, then $t_{1}^{*}$ must satisfy

$$
\begin{aligned}
& r c^{*}-\left[u\left(i_{0}, a_{1}^{*}\right)-u\left(i_{0}, a_{0}\right)\right]= \\
& h^{-}\left(t_{1}^{*}\right)\left(\frac{1}{r} u\left(i_{0}, a_{1}^{*}\right)\left(1-\gamma\left(t_{1}^{*}\right)\right)+\left[\gamma\left(t_{1}^{*}\right) \mathbb{E}^{+} V^{*}\left(I_{1}, a_{1}^{*}\right)-\mathbb{E}^{-} V^{*}\left(I_{1}, a_{0}\right)\right]-c^{*}\right)
\end{aligned}
$$

where $\gamma\left(t_{1}^{*}\right):=\frac{h^{+}\left(t_{1}^{*}\right)}{h^{-}\left(t_{1}^{*}\right)}$. Further, if the term multiplying $h^{-}\left(t_{1}^{*}\right)$ in $(37)$ is positive (resp. negative), then the second order conditions are strictly satisfied iff the hazard rate $h^{-}$is increasing (resp. decreasing) at $t_{1}^{*}$.

The arguments directly parallel the analysis for the innoculation model but is a good bit more involved because flow utilities change with change of action and arrival of the new state delivers the expected value of $V^{*}\left(I_{1}, a\right)$ where $a$ is the current action and expectations are taken with respect to the current conditional state change probability. These complications introduce several additional terms.

Throughout, we make use of two simplified versions of Leibniz's formula,

$$
\frac{d}{d t} \int_{t}^{\infty} h(x, t) d x=\int_{t}^{\infty} \frac{\partial h(x, t)}{\partial t} d x-h(t, t) \text { and } \frac{d}{d t} \int_{0}^{t} h(x, t) d x=\int_{0}^{t} \frac{\partial h(x, t)}{\partial t} d x+h(t, t) .
$$

4.1. Three Types of Events Plus Costs. There are three types of events with probability affected by the choice of $t_{1}$,

(A) $W=w$ and $0 \leq w<t_{1}$,

(B) $W=w$ and $t_{1} \leq w<\infty$,

(C) $W=w$ and $w=\infty$. 
If (A) happens, payoffs are $\int_{0}^{w} u\left(i_{0}, a_{0}\right) e^{-r x} d x+e^{-r w} \int_{S} V^{*}\left(j, a_{0}\right) \mu^{-}(w)(d j)$. Integrating over all such $w$ weighted with the likelihood $f^{-}(w)$ yields expected payoffs

$$
\int_{0}^{t_{1}}\left[\int_{0}^{w} u\left(i_{0}, a_{0}\right) e^{-r x} d x+e^{-r w} \int_{S} V^{*}\left(j, a_{0}\right) \mu^{-}(w)(d j)\right] f^{-}(w) d w .
$$

Using $\int_{0}^{t_{1}} e^{-r x} d x=\frac{1}{r}\left(1-e^{-r t_{1}}\right)$, the derivative of these payoffs with respect to $t_{1}$ is

$$
u\left(i_{0}, a_{0}\right) \frac{1}{r}\left(1-e^{-r t_{1}}\right) f^{-}\left(t_{1}\right)+e^{-r t_{1}} \mathbb{E}^{-} V^{*}\left(I_{1}, a_{0}\right) f^{-}\left(t_{1}\right)
$$

because progressivity guarantees that $\frac{\partial}{1}\left[\int_{S} V^{*}\left(j, a_{0}\right) \mu^{-}(w)(d j)\right] f^{-}(w)=0$

If (B) happens, payoffs are

$$
M\left(w, t_{1}\right):=u\left(i_{0}, a_{0}\right) \frac{1}{r}\left(1-e^{-r t_{1}}\right)+\int_{t_{1}}^{w} u\left(i_{0}, a_{1}^{*}\right) e^{-r x} d x+e^{-r w} \int_{S} V^{*}\left(j, a_{1}^{*}\right) \mu^{+}(w)(d j) .
$$

Integrating over all such $w$ weighted with the likelihood $f^{+}(w)$ yields expected payoffs $\int_{t_{1}}^{\infty} M\left(w, t_{1}\right) f^{+}(w) d w$ and the derivative of these payoffs with respect to $t_{1}$ is

$$
\begin{gathered}
-\left[\frac{1}{r}\left(1-e^{-r t_{1}}\right) u\left(i_{0}, a_{0}\right)+0+e^{-r t_{1}} \mathbb{E}^{+} V^{*}\left(I_{1}, a_{1}^{*}\right)\right] f^{+}\left(t_{1}\right) \\
+\frac{1}{r} e^{-r t_{1}}\left[u\left(i_{0}, a_{0}\right)-u\left(i_{0}, a_{1}^{*}\right)\right] \int_{t_{1}}^{\infty} f^{+}(w) d w
\end{gathered}
$$

plus the extra term

$$
\int_{t_{1}}^{\infty} e^{-r w} \frac{\partial}{\partial t_{1}}\left[\int_{S} V^{*}\left(j, a_{0}\right) \mu^{+}(w)(d j) f^{+}(w)\right] d w .
$$

For Theorem 2, this extra term is 0 because $\mu^{+}(w)$ and $f^{+}(w)$ are independent of $t_{1}<w$. In the next subsection, we will treat the extra terms.

If (C) happens, we define the incompleteness at $t_{1}$ as

$$
q\left(t_{1}\right)=\left(1-\left[\int_{0}^{t_{1}} f^{-}(w) d w+\int_{t_{1}}^{\infty} f^{+}(w) d w\right]\right)
$$

and define

$$
\begin{aligned}
z\left(t_{1}\right) & =\left[\int_{0}^{t_{1}} u\left(i_{0}, a_{0}\right) e^{-r x} d x+\int_{t_{1}}^{\infty} u\left(i_{0}, a_{1}^{*}\right) e^{-r x} d x\right] \\
& =\frac{1}{r}\left[u\left(i_{0}, a_{0}\right)\left(1-e^{-r t_{1}}\right)+u\left(i_{0}, a_{1}^{*}\right) e^{-r t_{1}}\right]
\end{aligned}
$$

so that the expected payoffs are $q\left(t_{1}\right) z\left(t_{1}\right)$. The derivative is $q^{\prime}\left(t_{1}\right) z\left(t_{1}\right)+q\left(t_{1}\right) z^{\prime}\left(t_{1}\right)$ where $z^{\prime}\left(t_{1}\right)=\left[u\left(i_{0}, a_{0}\right)-u\left(i_{0}, a_{1}^{*}\right)\right] e^{-r t_{1}}$ and $q^{\prime}\left(t_{1}\right)=\left[f^{+}\left(t_{1}\right)-f^{-}\left(t_{1}\right)\right]$ plus the extra term

$$
\int_{t_{1}}^{\infty} \frac{\partial}{\partial t_{1}} f^{+}(w) d w
$$

For Theorem 2, this extra term is 0 because $f^{+}(w)$ is independent of $t_{1}<w$. In the next subsection, we will treat this term in more generality. 
Combining, the derivative for case $(\mathrm{C})$ is

$$
\begin{aligned}
& {\left[f^{+}\left(t_{1}\right)-f^{-}\left(t_{1}\right)\right] \frac{1}{r}\left[u\left(i_{0}, a_{0}\right)\left(1-e^{-r t_{1}}\right)+u\left(i_{0}, a_{1}^{*}\right) e^{-r t_{1}}\right]} \\
& \quad+\left(1-\left[\int_{0}^{t_{1}} f^{-}(w) d w+\int_{t_{1}}^{\infty} f^{+}(w) d w\right]\right) \cdot\left[u\left(i_{0}, a_{0}\right)-u\left(i_{0}, a_{1}^{*}\right)\right] e^{-r t_{1}} .
\end{aligned}
$$

Expected costs add $-c^{*} e^{-r t_{1}}\left(1-F\left(t_{1}\right)\right)$ to the payoffs, and the marginal benefit of delay is the strictly positive

$$
\left[c^{*} e^{-r t_{1}} f^{-}\left(t_{1}\right)+\left(1-F\left(t_{1}\right)\right) r c^{*} e^{-r t_{1}}\right] .
$$

4.2. The FOCs. We now add all of the previous pieces of the derivative, (39), (40), (45) and (46), set the sum equal to 0 , and re-arrange. For organizing this, we gather the pieces are in the following table.

\begin{tabular}{lr|l} 
& & Table 1 \\
\hline \hline$(39)$ & (a) & $f^{-}\left(t_{1}\right) \cdot\left(e^{-r t_{1}} \mathbb{E}^{-} V^{*}\left(I, a_{0}\right)\right)$ \\
$(39)$ & (b) & $f^{-}\left(t_{1}\right) \cdot\left(u\left(i_{0}, a_{0}\right) \frac{1}{r}\left(1-e^{-r t_{1}}\right)\right)$ \\
\hline$(40)$ & (c) & $f^{+}\left(t_{1}\right) \cdot\left(-e^{-r t_{1}} \mathbb{E}^{+} V^{*}\left(I, a_{1}^{*}\right)\right)$ \\
$(40)$ & $(\mathrm{d})$ & $f^{+}\left(t_{1}\right) \cdot\left(-u\left(i_{0}, a_{0}\right) \frac{1}{r}\left(1-e^{-r t_{1}}\right)\right)$ \\
$(40)$ & $(\mathrm{e})$ & $\left(\int_{t_{1}}^{\infty} f^{+}(w) d w\right) \cdot e^{-r t_{1}}\left[u\left(i_{0}, a_{0}\right)-u\left(i_{0}, a_{1}^{*}\right)\right]$ \\
\hline$(45)$ & $(\mathrm{f})$ & $f^{+}\left(t_{1}\right) \cdot\left(u\left(i_{0}, a_{0}\right) \frac{1}{r}\left(1-e^{-r t_{1}}\right)\right)$ \\
$(45)$ & $\left(\mathrm{f}^{\prime}\right)$ & $f^{-}\left(t_{1}\right) \cdot\left(-u\left(i_{0}, a_{0}\right) \frac{1}{r}\left(1-e^{-r t_{1}}\right)\right)$ \\
$(45)$ & $(\mathrm{g})$ & $f^{+}\left(t_{1}\right) \cdot\left(u\left(i_{0}, a_{1}^{*}\right) \frac{1}{r} e^{-r t_{1}}\right)$ \\
$(45)$ & $\left(\mathrm{g}^{\prime}\right)$ & $f^{-}\left(t_{1}\right) \cdot\left(-u\left(i_{0}, a_{1}^{*}\right) \frac{1}{r} e^{-r t_{1}}\right)$ \\
$(45)$ & $(\mathrm{h})$ & $\left(1-\left[\int_{0}^{t_{1}} f^{-}(w) d w+\int_{t_{1}}^{\infty} f^{+}(w) d w\right]\right) \cdot e^{-r t_{1}}\left[u\left(i_{0}, a_{0}\right)-u\left(i_{0}, a_{1}^{*}\right)\right]$ \\
\hline$(46)$ & $(\mathrm{i})$ & $f^{-}\left(t_{1}\right) \cdot c^{*} e^{-r t_{1}}$ \\
$(46)$ & $(\mathrm{j})$ & $\left(1-\int_{0}^{t_{1}} f^{-}(w) d w\right) \cdot r c^{*} e^{-r t_{1}}$
\end{tabular}

First observe that the term $u\left(i_{0}, a_{0}\right) \frac{1}{r}\left(1-e^{-r t_{1}}\right)$ appears four places, in (b), (d), (f), and $\left(f^{\prime}\right)$, and these four terms cancel. Second, observe that every term remaining in the table has a common factor of $e^{-r t_{1}}$. Third, the terms (e), (h) and (j) sum to $e^{-r t_{1}}$ times

$$
\left(1-F\left(t_{1}\right)\right)\left(r c^{*}-\left[u\left(i_{0}, a_{1}^{*}\right)-u\left(i_{0}, a_{0}\right)\right]\right) .
$$

Taking out the lines (b), (d), (e), (f), ( $\left.\mathrm{f}^{\prime}\right),(\mathrm{h})$ and $(\mathrm{j})$ and removing the common factor of $e^{-r t_{1}}$ from the remaining lines yields the following reduced table. 


\begin{tabular}{lr|l} 
& & Table 1 (reduced) \\
\hline \hline$(39)$ & $(\mathrm{a})$ & $f^{-}\left(t_{1}\right) \cdot\left(\mathbb{E}^{-} V^{*}\left(I, a_{0}\right)\right)$ \\
\hline$(40)$ & $(\mathrm{c})$ & $f^{+}\left(t_{1}\right) \cdot\left(-\mathbb{E}^{+} V^{*}\left(I, a_{1}^{*}\right)\right)$ \\
\hline$(45)$ & $(\mathrm{g})$ & $f^{+}\left(t_{1}\right) \cdot\left(u\left(i_{0}, a_{1}^{*}\right) \frac{1}{r}\right)$ \\
$(45)$ & $\left(\mathrm{g}^{\prime}\right)$ & $f^{-}\left(t_{1}\right) \cdot\left(-u\left(i_{0}, a_{1}^{*}\right) \frac{1}{r}\right)$ \\
\hline$(46)$ & $(\mathrm{i})$ & $f^{-}\left(t_{1}\right) \cdot c^{*}$
\end{tabular}

The five terms in this table sum to

$$
\begin{aligned}
& \frac{1}{r} u\left(i_{0}, a_{1}^{*}\right)\left(f^{+}\left(t_{1}\right)-f^{-}\left(t_{1}\right)\right)+ \\
& \quad\left[f^{-}\left(t_{1}\right) \cdot \mathbb{E}^{-} V^{*}\left(I, a_{0}\right)-f^{+}\left(t_{1}\right) \cdot \mathbb{E}^{+} V^{*}\left(I, a_{1}^{*}\right)\right]+f^{-}\left(t_{1}\right) c^{*}
\end{aligned}
$$

Letting $\gamma(t)=\frac{f^{+}(t)}{f^{-}(t)}=\frac{h^{+}(t)}{h^{-}(t)},(48)$ simplifies to

$$
f^{-}\left(t_{1}\right)\left(\frac{1}{r} u\left(i_{0}, a_{1}^{*}\right)\left(\gamma\left(t_{1}\right)-1\right)+\left[\mathbb{E}^{-} V^{*}\left(I, a_{0}\right)-\gamma\left(t_{1}\right) \mathbb{E}^{+} V^{*}\left(I, a_{1}^{*}\right)\right]+c^{*}\right) .
$$

Adding (47) and (49) and setting the sum equal to 0 and dividing both sides by $\left(1-F\left(t_{1}\right)\right)$ yields

$$
\begin{aligned}
& \left(r c^{*}-\left[u\left(i_{0}, a_{1}^{*}\right)-u\left(i_{0}, a_{0}\right)\right]\right)= \\
& \quad h^{-}\left(t_{1}\right)\left(\frac{1}{r} u\left(i_{0}, a_{1}^{*}\right)\left(1-\gamma\left(t_{1}\right)\right)+\left[\gamma\left(t_{1}\right) \mathbb{E}^{+} V^{*}\left(I, a_{1}^{*}\right)-\mathbb{E}^{-} V^{*}\left(I, a_{0}\right)\right]-c^{*}\right),
\end{aligned}
$$

the FOCs of Theorem 2.

4.3. FOCs for a More General Problem. For Theorem 2, we assumed that for $t_{1}<$ $t_{1}^{\prime}<w, \mu\left(w, t_{1}\right)=\mu\left(w, t_{1}^{\prime}\right)=\mu^{+}(w)$ and $f\left(w, t_{1}\right)=f\left(w, t_{1}^{\prime}\right)=f^{+}(w)$ for a pair $\mu^{+}(\cdot)$ and $f^{+}(\cdot)$ that depend on the chosen $a_{1}^{*}$. We now complete the analysis, begun in equations (41) and (44), of the extra terms that arise when these assumptions are not true. We will see that there are two additional terms in the FOCs: one involving the expected discounted change in the value of the new state-action pair that arises from $t_{1}$ 's effect on the discounted future values of $\left[\mu\left(w, t_{1}\right) f\left(w, t_{1}\right)\right]$; and one involving the annuitized value of expected discounted flow losses that arise from $t_{1}$ 's effect on the discounted value of $f\left(w, t_{1}\right)$.

For ease in readibility only, we assume that $S$ is discrete so that " $\int_{S} v(j) \mu\left(w, t_{1}\right)(d j)$ " can be replaced by " $\sum_{j} v(j) \mu_{j}\left(w, t_{1}\right) . "$

Without the simplifying assumptions, there should be four extra lines for Table 1 from equation (40), one involving the partial derivative of $\mu\left(w, t_{1}\right)$ with respect to $t_{1}$ and three involving the partial derivative of $f\left(w, t_{1}\right)$ with respect to $t_{1}$. 


\begin{tabular}{ll|l} 
& & Table $(\mathrm{B})$ \\
\hline \hline$(40)$ & $(\mathrm{x} 1)$ & $\sum_{j} V^{*}\left(j, a_{1}^{*}\right) \int_{t_{1}}^{\infty} e^{-r w} \frac{\partial \mu_{j}\left(w, t_{1}\right)}{\partial t_{1}} f\left(w, t_{1}\right) d w$ \\
\hline$(40)$ & $(\mathrm{x} 2)$ & $u\left(i_{0}, a_{0}\right) \frac{1}{r}\left(1-e^{-r t_{1}}\right) \cdot \int_{t_{1}}^{\infty} \frac{\partial f\left(w, t_{1}\right)}{\partial t_{1}} d w$ \\
$(40)$ & $(\mathrm{x} 3)$ & $u\left(i_{0}, a_{1}^{*}\right) \frac{1}{r} e^{-r t_{1}} \cdot \int_{t_{1}}^{\infty}\left(1-e^{-r\left(w-t_{1}\right)}\right) \frac{\partial f\left(w, t_{1}\right)}{\partial t_{1}} d w$ \\
$(40)$ & $(\mathrm{x} 4)$ & $\sum_{j} V^{*}\left(j, a_{1}^{*}\right) \int_{t_{1}}^{\infty} e^{-r w} \mu_{j}\left(w, t_{1}\right) \frac{\partial f\left(w, t_{1}\right)}{\partial t_{1}} d w$
\end{tabular}

Without the simplifying assumptions, there is an extra term to $q^{\prime}\left(t_{1}\right)$, hence an extra pair of terms to $q^{\prime}\left(t_{1}\right) z\left(t_{1}\right)$ in (45).

\begin{tabular}{ll|l} 
& & Table $(\mathrm{C})$ \\
\hline \hline$(45)$ & $(\mathrm{x} 1)$ & $u\left(i_{0}, a_{0}\right) \frac{1}{r}\left(1-e^{-r t_{1}}\right) \cdot\left(-\int_{t_{1}}^{\infty} \frac{\partial f\left(w, t_{1}\right)}{\partial t_{1}} d w\right)$ \\
$(45)$ & $(\mathrm{x} 2)$ & $u\left(i_{0}, a_{1}^{*}\right) \frac{1}{r} e^{-r t_{1}} \cdot\left(-\int_{t_{1}}^{\infty} \frac{\partial f\left(w, t_{1}\right)}{\partial t_{1}} d w\right)$
\end{tabular}

The FOCs for the more general problem involve the sum of the four terms in Table (B) and the two in Table (C). (40)(x2) and (45)(x1) cancel, and (40)(x1) plus (40)(x4) can be re-written as $\sum_{j} V^{*}\left(j, a_{1}^{*}\right) e^{-r t_{1}} \int_{t_{1}}^{\infty} e^{-r\left(w-t_{1}\right)} \frac{\partial\left[\mu_{j}\left(w, t_{1}\right) f\left(w, t_{1}\right)\right]}{\partial t_{1}} d w$, yielding the following reduced table.

\begin{tabular}{rr|l} 
& & Tables $(\mathrm{B})$ and $(\mathrm{C})$ reduced \\
\hline \hline$(40)$ & $(\mathrm{x} 1)+(\mathrm{x} 4)$ & $\sum_{j} V^{*}\left(j, a_{1}^{*}\right) e^{-r t_{1}} \int_{t_{1}}^{\infty} e^{-r\left(w-t_{1}\right)} \frac{\partial\left[\mu_{j}\left(w, t_{1}\right) f\left(w, t_{1}\right)\right]}{\partial t_{1}} d w$ \\
$(40)$ & $(\mathrm{x} 3)$ & $u\left(i_{0}, a_{1}^{*}\right) \frac{1}{r} e^{-r t_{1}} \cdot \int_{t_{1}}^{\infty}\left(1-e^{-r\left(w-t_{1}\right)}\right) \frac{\partial f\left(w, t_{1}\right)}{\partial t_{1}} d w$ \\
\hline$(45)$ & $(\mathrm{x} 2)$ & $u\left(i_{0}, a_{1}^{*}\right) \frac{1}{r} e^{-r t_{1}} \cdot\left(-\int_{t_{1}}^{\infty} \frac{\partial f\left(w, t_{1}\right)}{\partial t_{1}} d w\right)$
\end{tabular}

Cancelling out the common parts of $(40)(\mathrm{x} 3)$ and $(45)(\mathrm{x} 2)$, the FOCs for the more general version of Theorem 2 involve $e^{-r t_{1}}$ times two additional terms,

$$
\begin{aligned}
& \sum_{j} V^{*}\left(j, a_{1}^{*}\right) \int_{t_{1}}^{\infty} e^{-r\left(w-t_{1}\right)} \frac{\partial\left[\mu_{j}\left(w, t_{1}\right) f\left(w, t_{1}\right)\right]}{\partial t_{1}} d w, \text { and } \\
& -u\left(i_{0}, a_{1}^{*}\right) \frac{1}{r} \int_{t_{1}}^{\infty} e^{-r\left(w-t_{1}\right)} \frac{\partial f\left(w, t_{1}\right)}{\partial t_{1}} d w .
\end{aligned}
$$

The first term corresponds to the expected discounted change in the value of the new state-action pair that arises from $t_{1}$ 's effect on the distribution of future changes, the second corresponds to the annuitized value of expected discounted flow losses that arise from $t_{1}$ 's effect on the distribution of the future arrival of the state change.

\section{REFERENCES}

Corbae, D., M. Stinchcombe, and J. Zeman. 2009. An Introduction to Mathematical Analysis for Economic Theory and Econometrics. Princeton University Press.

Dudley, R. M. 2002. Real Analysis and Probability. Vol. 74 of Cambridge Studies in Advanced Mathematics, Cambridge:Cambridge University Press. Revised reprint of the 1989 original. 
Simon, Leo K., and Maxwell B. Stinchcombe. 1989. "Extensive Form games in Continuous Time: Pure strategies." Econometrica, 57(5): 1171-1214.

Stinchcombe, Maxwell B. 1992. "Maximal Strategy sets for Continuous-time Game Theory." Journal of Economic Theory, 56(2): 235-265. 